\title{
Morbidity and mortality in patients with hyperprolactinaemia: the PROLEARS study
}

\author{
Enrique Soto-Pedre', Paul J Newey ${ }^{1,2}$, John S Bevan ${ }^{3}$ and Graham P Leese ${ }^{1,2}$ \\ 'Division of Molecular and Clinical Medicine, School of Medicine, Ninewells Hospital and Medical School, \\ University of Dundee, Dundee, UK \\ 2Department of Endocrinology and Diabetes, Ninewells Hospital and Medical School, University of Dundee, \\ Dundee, UK \\ ${ }^{3}$ JJR Macleod Centre for Diabetes, Endocrinology and Metabolism (Mac-DEM), Aberdeen Royal Infirmary, \\ University of Aberdeen, Aberdeen, UK
}

Correspondence

should be addressed

to E Soto-Pedre

Email

e.soto@dundee.ac.uk

\begin{abstract}
Purpose: High serum prolactin concentrations have been associated with adverse health outcomes in some but not all studies. This study aimed to examine the morbidity and allcause mortality associated with hyperprolactinaemia.

Methods: A population-based matched cohort study in Tayside (Scotland, UK) from 1988 to 2014 was performed. Record-linkage technology was used to identify patients with hyperprolactinaemia that were compared to an age-sex-matched cohort of patients free of hyperprolactinaemia. The number of deaths and incident admissions with diabetes mellitus, cardiovascular disease, cancer, breast cancer, bone fractures and infectious conditions were compared by the survival analysis.

Results: Patients with hyperprolactinaemia related to pituitary tumours had no increased risk of diabetes, cardiovascular disease, bone fractures, all-cause cancer or breast cancer. Whilst no increased mortality was observed in patients with pituitary microadenomas $(\mathrm{HR}=1.65,95 \% \mathrm{Cl}: 0.79-3.44)$, other subgroups including those with pituitary macroadenomas and drug-induced and idiopathic hyperprolactinaemia demonstrated an increased risk of death. Individuals with drug-induced hyperprolactinaemia also demonstrated increased risks of diabetes, cardiovascular disease, infectious disease and bone fracture. However, these increased risks were not associated with the degree of serum prolactin elevation $\left(P_{\text {trend }}>0.3\right)$. No increased risk of cancer was observed in any subgroup.

Conclusions: No excess morbidity was observed in patients with raised prolactin due to pituitary tumours. Although the increased morbidity and mortality associated with defined patient subgroups are unlikely to be directly related to the elevation in serum prolactin, hyperprolactinaemia might act as a biomarker for the presence of some increased disease risk in these patients.
\end{abstract}

\author{
Key Words \\ - hyperprolactinaemia \\ - prolactin \\ - pituitary gland \\ - breast cancer \\ - mortality
}

Endocrine Connections (2017) 6, 580-588

\section{Introduction}

Hyperprolactinaemia is a common condition in endocrine practice (1). It may occur in men and women at any age, and its prevalence and incidence depend on the study population. Its epidemiology in the general population has been recently established by the Prolactin Epidemiology, Audit and Research Study (PROLEARS) a population-based cohort study in Tayside (Scotland, UK) (2). This study showed an overall prevalence of

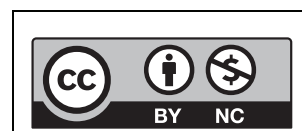

This work is licensed under a Creative Commons Attribution-NonCommercial 4.0 International License. 
hyperprolactinaemia (i.e. prolactin greater than $1000 \mathrm{U} / \mathrm{L}$ ) over a period of 20 years of 1 per 1000 of the population and the age-sex-adjusted incidence rates were 21.5 per 100,000 person-years for women and 6.4 per 100,000 person-years for men. The highest incidence rates were found in women aged 25-44 years.

Hyperprolactinaemia can be physiological, pathological or iatrogenic, and the causes of nonphysiological hyperprolactinaemia include pituitary disorders, drugs (most commonly antipsychotic agents) and hypothyroidism; aside from these, idiopathic hyperprolactinaemia is not uncommon (3). In the PROLEARS study, the commonest cause was druginduced (45.9\%), followed by pituitary disorders (25.4\%) and idiopathic cases (15\%) (2). Regardless of aetiology, hyperprolactinaemia may cause symptoms related to excess prolactin (e.g. galactorrhoea) or due to suppression of the hypothalamic-pituitary-gonadal axis (e.g. amenorrhea, infertility and erectile dysfunction). In addition, high concentrations of serum prolactin have been inconsistently related to several adverse health outcomes including cardiovascular disease, cancer, osteoporosis, autoimmune conditions and all-cause mortality (1).

There are little useful data on hyperprolactinaemia and cardiovascular endpoints. Controversy exists as to whether hyperprolactinaemia may be associated with heart valve disease (including that related to the use of dopamine agonist therapy), cardiovascular risk factors or cardiovascular mortality $(4,5,6,7)$. Results from epidemiologic studies that have examined the relationship between prolactin and all-cause cancer or breast cancer have also given inconsistent results $(8,9,10,11,12,13,14,15,16)$. Similarly, there are no consistent correlations reported between prolactin levels and bone loss or autoimmune diseases; thus, the clinical significance of hyperprolactinaemia in these patients remains unclear $(17,18,19,20,21,22,23,24,25,26)$. There are little available data on mortality associated with hyperprolactinaemia, although one study suggested that high serum prolactin levels might be associated with an increased risk of death (6). These results give grounds for a hypothesis that there may be subgroups of patients with hyperprolactinaemia at variable health risks.

The Centre for Endocrine Epidemiology in Tayside (Scotland, UK) has undertaken data linkage to report on incidence and prevalence of hyperprolactinaemia (2). We aimed to use the same data linkage to examine morbidity and mortality associated with hyperprolactinaemia, as well as the relative contribution of the respective causes of hyperprolactinaemia.

\section{Subjects and methods}

A population-based retrospective matched cohort study was performed among patients ever registered with a general practitioner in Tayside, a well-defined geographical region within Scotland (UK), with a mainly Caucasian population of 400,000 people. All patients are issued a unique patient identification number (Community Health Index) which facilitates the linkage of all electronic medical records and official registers. The biochemistry database was linked anonymously to other databases by the Health Informatics Centre Services/Farr Institute of Scotland at the University of Dundee (http:// www.dundee.ac.uk/hic) as previously described (2). These included demographic records (gender, birth, migration and deprivation based on patient's full postcode Scottish Index of Multiple Deprivation - SIMD), Scottish Morbidity Records - SMR (maternity admissions, hospital admissions, surgical procedures and cancer registration), Scottish Care Information-Diabetes Collaboration SCIDC database (data from primary care and diabetes clinics on diabetes mellitus), prescriptions dispensed and the General Registrar Office - GRO records on patient deaths. Magnetic Resonance Imaging (MRI) reports were also accessed and linked for identifying pituitary tumours. The International Classification of Diseases (ICD) 9-10th revision codes were used in the SMR to identify hospital inpatient and cancer events, and prescriptions were identified by means of the British National Formulary (BNF) codes (Table 1). To ensure data quality, SMR data are routinely subjected to a set of validation rules by the Information Services Division (ISD - NHS National Services Scotland) to evaluate and ensure that these datasets are accurate, consistent and comparable across time and between sources (27).

\section{Hyperprolactinaemia and comparison cohort definition}

The identification of individuals with hyperprolactinaemia among those with at least one measurement of a serum prolactin level taken was as previously reported (2). Any patient with a serum prolactin measurement greater than $1000 \mathrm{U} / \mathrm{L}(47.2 \mathrm{ng} / \mathrm{mL})$ and/or three or more prescriptions of specific dopamine agonists (BNF 6.7.1: cabergoline, http://www.endocrineconnections.org DOI: 10.1530/EC-17-0171 (c) 2017 The authors Published by Bioscientifica Ltd
This work is licensed under a Creative Commons Attribution-NonCommercial 4.0 International License. 
Table 1 Codes used to identify hospital admissions, cancer registration and prescriptions of interest.

\begin{tabular}{l} 
Description \\
\hline Admissions (SMR) \\
Bone fractures \\
Osteoporotic bone fractures \\
Cardiovascular disease \\
Cancer \\
Breast cancer \\
Infectious conditions
\end{tabular}

Autoimmune conditions

\section{Prescriptions}

Prednisolone

Bisphosphonates

Dopamine agonists

HRT

\begin{tabular}{l}
\hline \\
ICD-9 codes \\
$800-829$, E887 \\
$733.1,805.4,805.5,806.4,806.5,813,820,821$ \\
$410-429$ \\
$140-209$ \\
$174,175,198.81,233.0$ \\
$001-136,460-466,480-487,680-686$
\end{tabular}

242.0, 245.8, 255.4, 258.1, 579.0, 340, 358,

571.4, 571.6, 695.4, 710.2, 710.3, 710.4

BNF codes

within 6.3.2

6.6 .2

6.7 .1

7.3.1

\section{ICD-10 codes} \\ M484, M80, M81, S02, S12, S22, S32, S42, S52, S62, \\ S72, S82, S92, T02, T08, T10, T12, T142 \\ $\mathrm{M} 80, \mathrm{S32}, \mathrm{S} 52, \mathrm{~S} 72$ \\ $120-125,144,145,147-150$ \\ C00-C97; D00-D09 \\ C50, D05 \\ A00-A99, B00-B99, G00-G02, G04-G07, J00-J22, \\ L00-L08 \\ D51.0, E27.1, E27.2, E31.0, E03.5, E03.8, E05.0, \\ E06.3, G70.0, K74.3, K90.0, M32-M35
}

BNF, British National Formulary; HRT, Oestrogen containing hormone replacement therapy; ICD, International Classification of Diseases (ICD-9=Ninth Revision codes, ICD-10= Tenth Revision codes); SMR, Scottish Morbidity Records.

bromocriptine and/or quinagolide) during the follow-up period was considered as exposed, with subsequent allocation to one of the four cause-related subgroups as follows:

1. Pituitary disorder. Patients were selected if they had at least one serum prolactin measurement greater than $5000 \mathrm{U} / \mathrm{L}(235.8 \mathrm{ng} / \mathrm{mL})$, and/or three or more prescriptions of specific dopamine agonists, hospital admissions related to pituitary disorder (ICD10: C751, E22, E23), pituitary surgery (OPCS-4: B01, B02, B04) or a mass on pituitary MRI.

2. Drug-induced. Patients were selected if they had a record of being prescribed $\operatorname{drug}(\mathrm{s})$ known to elevate prolactin levels within 6 months before and 1 month after the assessment of serum prolactin measurement, and did not fit into pituitary disorder group. Any prescription of antipsychotics (BNF: 4.2.1, 4.2.2), tricyclics and related antidepressants (BNF: 4.3.1), serotonin re-uptake inhibitors (BNF: 4.3.3), dopamine antagonists (BNF: 4.6), opioids and drugs used in opioid dependence (BNF: 4.7.2, 4.10.3), $\mathrm{H}_{2}$ antagonists (BNF: 1.3.1), methyldopa (in BNF: 2.5.2) and verapamil (in BNF: 2.6.2) was extracted from the database containing prescriptions dispensed from all community pharmacies in Tayside.

3. Macroprolactin. Patients were selected if they had a presence of macroprolactin identified without any other explanation.

4. Hypothyroidism. Patients were selected if they had a thyroid-stimulating hormone (TSH) serum level $>6 \mathrm{U} / \mathrm{L}(0.28 \mathrm{ng} / \mathrm{mL})$ at some time without any other explanation. (c) 2017 The authors Published by Bioscientifica Ltd
5. Idiopathic. Patients were selected if remained unclassified.

The pituitary disorder group was further stratified into micro- $(\leq 1 \mathrm{~cm})$ and macroadenoma $(>1 \mathrm{~cm})$ subgroups based on available MRI reports. All patients who were pregnant or possibly lactating at the time of assay (i.e. within 9 months before and 12 months after the delivery date) were excluded (unless they had raised prolactin measurements out with this time window) together with those patients with hyperprolactinaemia related to macroprolactin.

For each patient with hyperprolactinaemia, the patients' register was used to identify five patients matched by age ( \pm 6 months) and gender for comparison, that were free of hyperprolactinaemia (i.e. unexposed). Baseline data at the date of entry into the study were collected for all patients. The numbers of patients with defined clinical outcomes of interest before and after entering the study were also identified. The period of follow-up for the analysis was defined from 1988 to 2014. Each eligible patient was followed up until either occurrence of outcome of interest or end of study (i.e. moving out of health area, death or 30 May 2014).

\section{Statistical analysis}

The mean and standard deviation or the median and the interquartile range of the data were calculated to describe continuous variables. ANOVA and chi-square tests were used to compare means and frequencies among subgroups of patients, respectively. Non-parametric methods were used where appropriate.

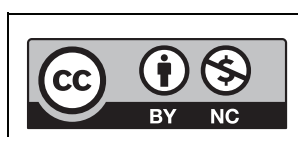

This work is licensed under a Creative Commons Attribution-NonCommercial 4.0 International License. 
Survival analysis was performed to follow-up patients and Cox proportional hazards' models using the robust variance estimator were used to explore the relationship between exposure to hyperprolactinaemia and clinical outcomes. A separate analysis estimating the hazard ratios (HRs) with 95\% confidence intervals (CI) associated with hyperprolactinaemia was performed for each outcome. Log-rank tests of equality across strata were obtained for all categorical covariates, and univariate Cox models were fitted for the continuous covariates to explore whether or not to also include them in the multivariate model. Categorical covariates were included as dummy variables. Adjusted models were stratified by the matched sets of subjects on age and gender (i.e. matched analysis). A test of the proportional hazards was performed for each covariate and globally using a formal significance test based on Schoenfeld residuals and by plotting log-log plots (28). Model specification was evaluated using goodness-offit diagnostics by computing Harrell's C coefficient (28). Exposure was coded as binary (i.e. ever vs never had hyperprolactinaemia) as well as dummy variables to consider six levels of exposure (i.e. never had hyperprolactinaemia, ever had hyperprolactinaemia related to a pituitary microadenoma, pituitary macroadenoma, drug-induced, secondary to hypothyroidism or idiopathic causes). Quintiles of the serum prolactin concentration were used to break the range of the exposure into categories and look for trends in the category-specific HRs (i.e. dose-response or trend analysis). Data were entered into a STATA/MP version 14.0 software package (StataCorp, USA) for statistical analysis and determination of statistical significance $(P<0.05)$.

\section{Outcomes}

All outcomes were incident and coded as binary. Patients with prevalent outcomes of interest at baseline were excluded from the survival analysis of that particular outcome. Outcomes were death, diabetes mellitus, bone fractures, non-fatal cardiovascular disease, cancer, autoimmune conditions and infectious conditions. Limiting this study to only incident outcomes avoided bias from missing data on prior events (i.e. left censoring) (29).

For some outcomes being modelled, the following covariates were also considered for inclusion: history of medication used, history of renal impairment (serum

Table 2 Description of patients at first diagnosis of hyperprolactinaemia and their comparison cohort.

\begin{tabular}{|c|c|c|c|}
\hline Characteristic & Hyperprolactinaemia $(n=1204)$ & Comparison cohort $(n=5888)$ & $\boldsymbol{P}$ \\
\hline \multicolumn{4}{|l|}{$n(\%)$} \\
\hline Gender- female & $943(78.3)$ & $4602(78.2)$ & $=0.901$ \\
\hline \multicolumn{4}{|l|}{ SIMD quintile } \\
\hline 1 most deprived & $309(26.4)$ & $1162(20.5)$ & \\
\hline 2 & $230(19.7)$ & 995 (17.6) & \\
\hline 3 & $184(15.7)$ & $926(16.4)$ & \\
\hline 4 & $306(26.2)$ & 1634 (28.9) & \\
\hline 5 most affluent & $141(12.1)$ & $940(16.3)$ & $<0.001$ \\
\hline Diabetes Mellitus & $70(5.8)$ & $204(3.5)$ & $<0.001$ \\
\hline Non-fatal Cardiovascular disease & $62(5.2)$ & $183(3.1)$ & $<0.001$ \\
\hline Cancer & $101(8.4)$ & $411(6.9)$ & $=0.085$ \\
\hline Bone fractures & $76(6.3)$ & $264(4.5)$ & $=0.007$ \\
\hline Infectious conditions & $198(16.5)$ & $588(9.9)$ & $<0.001$ \\
\hline Renal impairment & $79(6.5)$ & $378(6.4)$ & $=0.855$ \\
\hline \multicolumn{4}{|l|}{ Medication use } \\
\hline Bisphosphonate & $16(1.3)$ & $62(1.1)$ & $=0.403$ \\
\hline Prednisolone & $93(7.7)$ & $282(4.8)$ & $<0.001$ \\
\hline HRT & $205(17.0)$ & $1270(21.6)$ & $<0.001$ \\
\hline \multicolumn{4}{|l|}{ Mean (s.D.) } \\
\hline Age (years)* & $39.5(16.5)$ & $38.7(15.6)$ & $=0.123$ \\
\hline Serum prolactin (U/L) & $1514(1177-2382)$ & 193 (143-255) & $<0.001$ \\
\hline Serum TSH (U/L) $\phi$ & $2.2(1.4-3.9)$ & $2.4(1.6-3.5)$ & $=0.003$ \\
\hline
\end{tabular}

$\left.{ }^{*}\right)$ Age at study entry $(\phi)$ Maximum serum concentration: median (interquartile range).

HRT, Oestrogen containing hormone replacement therapy; SIMD, Scottish Index of Multiple Deprivation; TSH, Thyroid-stimulating hormone.
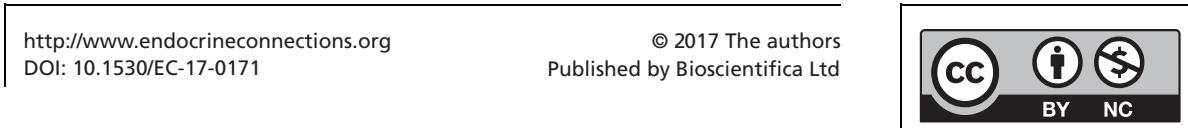

This work is licensed under a Creative Commons Attribution-NonCommercial 4.0 International License. 
Table 3 Estimates of hazard ratios for hyperprolactinaemia on having several clinical outcomes by related cause of hyperprolactinaemia.

\begin{tabular}{|c|c|c|c|c|c|c|}
\hline \multirow[b]{2}{*}{ Outcome } & \multirow[b]{2}{*}{ Time at risk (years) } & \multirow[b]{2}{*}{ Events $n$} & \multicolumn{2}{|c|}{ Unadjusted } & \multicolumn{2}{|c|}{ Adjusted } \\
\hline & & & $\mathrm{HR}$ & $95 \% \mathrm{Cl}$ & $\mathrm{HR}$ & $95 \% \mathrm{Cl}$ \\
\hline Diabetes mellitus & 64,068 & 334 & & & & \\
\hline Microadenoma & & & 0.885 & $0.461-1.699$ & 1.203 & $0.643-2.250$ \\
\hline Macroadenoma & & & 1.925 & $0.710-5.220$ & 1.419 & $0.542-3.714$ \\
\hline Drug-induced & & & 2.032 & $1.408-2.932 *$ & 1.638 & $1.154-2.325 *$ \\
\hline Hypothyroidism & & & 0.884 & $0.364-2.148$ & 1.431 & $0.555-3.689$ \\
\hline Idiopathic & & & 0.716 & $0.352-1.456$ & 0.898 & $0.452-1.785$ \\
\hline Non-fatal Cardiovascular disease ${ }^{a}$ & 64,805 & 238 & & & & \\
\hline Microadenoma & & & 0.698 & $0.287-1.700$ & 1.383 & $0.543-3.519$ \\
\hline Macroadenoma & & & 2.641 & $0.977-7.137$ & 2.183 & $0.863-5.524$ \\
\hline Drug-induced & & & 1.758 & $1.128-2.739 *$ & 1.854 & $1.171-2.937 *$ \\
\hline Hypothyroidism & & & 1.544 & $0.669-3.560$ & 2.071 & $0.846-5.064$ \\
\hline Idiopathic & & & 0.651 & $0.266-1.591$ & 1.222 & $0.506-2.949$ \\
\hline Cancer & 61,714 & 440 & & & & \\
\hline Microadenoma & & & 0.877 & $0.492-1.563$ & 1.447 & $0.821-2.549$ \\
\hline Macroadenoma & & & 2.087 & $0.919-4.738$ & 1.580 & $0.628-3.976$ \\
\hline Drug-induced & & & 1.370 & $0.956-1.962$ & 0.977 & $0.668-1.427$ \\
\hline Hypothyroidism & & & 0.945 & $0.447-1.998$ & 1.487 & $0.746-2.963$ \\
\hline Idiopathic & & & 0.614 & $0.317-1.189$ & 0.811 & $0.413-1.593$ \\
\hline Bone fractures $^{b}$ & 63,627 & 285 & & & & \\
\hline Microadenoma & & & 0.847 & $0.401-1.791$ & 1.435 & $0.636-3.238$ \\
\hline Macroadenoma & & & 0.534 & $0.075-3.802$ & 0.664 & $0.120-3.667$ \\
\hline Drug-induced & & & 2.733 & $1.932-3.865 *$ & 2.087 & $1.478-2.948 *$ \\
\hline Hypothyroidism & & & 1.103 & $0.443-2.746$ & 1.219 & $0.491-3.025$ \\
\hline Idiopathic & & & 0.911 & $0.449-1.849$ & 1.190 & $0.571-2.481$ \\
\hline Infectious conditions ${ }^{c}$ & 59,231 & 623 & & & & \\
\hline Microadenoma & & & 0.619 & $0.339-1.132$ & 0.727 & $0.420-1.259$ \\
\hline Macroadenoma & & & 1.849 & $0.929-3.679$ & 1.245 & $0.602-2.576$ \\
\hline Drug-induced & & & 2.276 & $1.771-2.925 *$ & 1.899 & $1.479-2.440 *$ \\
\hline Hypothyroidism & & & 1.339 & $0.772-2.323$ & 1.296 & $0.740-2.269$ \\
\hline Idiopathic & & & 0.622 & $0.348-1.111$ & 0.848 & $0.482-1.491$ \\
\hline Death $^{d}$ & 65,579 & 465 & & & & \\
\hline Microadenoma & & & 0.886 & $0.471-1.669$ & 1.658 & $0.798-3.446$ \\
\hline Macroadenoma & & & 6.207 & 3.694-10.427* & 2.815 & $1.420-5.579 *$ \\
\hline Drug-induced & & & 4.164 & $3.276-5.293 *$ & 3.358 & $2.568-4.392 *$ \\
\hline Hypothyroidism & & & 2.680 & $1.606-4.472 *$ & 5.125 & $2.457-10.691$ * \\
\hline Idiopathic & & & 1.954 & $1.294-2.951$ * & 3.728 & $2.306-6.027 *$ \\
\hline
\end{tabular}

Adjusted models were stratified by the matched sets of subjects on age and gender. $\left(^{*}\right) P<0.05$.

aAdjusted also for history of diabetes mellitus and renal impairment.

${ }^{\text {b} A d j u s t e d ~ a l s o ~ f o r ~ h i s t o r y ~ o f ~ b i s p h o s p h o n a t e s ~ u s e, ~ p r e d n i s o l o n e ~ u s e ~ a n d ~ r e n a l ~ i m p a i r m e n t . ~}$

'Adjusted also for history of prednisolone use.

${ }^{\mathrm{d} A d j u s t e d}$ also for history of non-fatal cardiovascular disease, renal impairment and a Scottish index of multiple deprivation.

HR, Hazard ratio.

creatinine level $>150 / 120 \mu \mathrm{mol} / \mathrm{L}$ for men/ women, respectively) and social deprivation index - SIMD.

\section{Ethical approval}

All analyses were performed on anonymised datasets. The study was approved by the Tayside Medical Ethics Committee and data protection by the Tayside Caldicott Guardians, and conducted in accordance with the
Declaration of Helsinki. The funders had no role in design, analysis, interpretation or writing of the manuscript.

\section{Results}

We identified 1204 patients with hyperprolactinaemia (331 related to a pituitary disorder, 598 drug-induced, 79 secondary to hypothyroidism and 196 idiopathic causes) and 5888 age- and sex-matched patients as a comparison

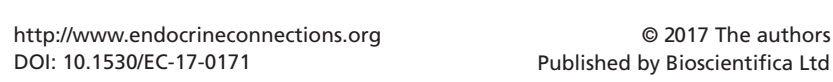


A

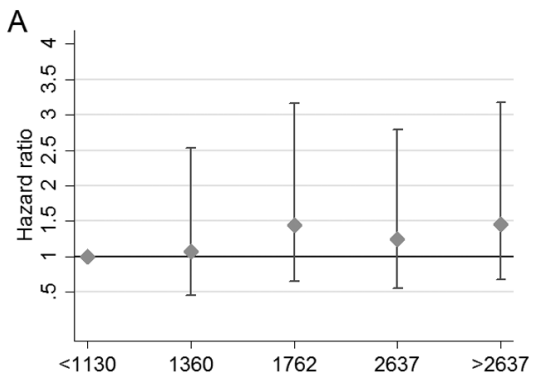

C

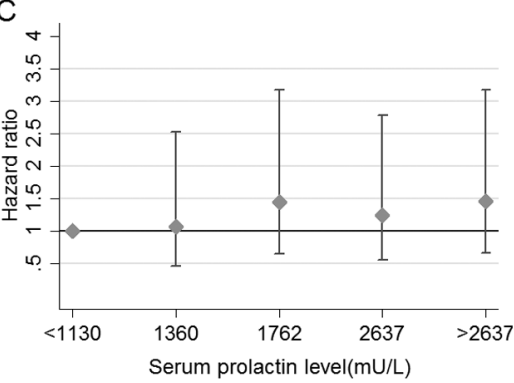

B

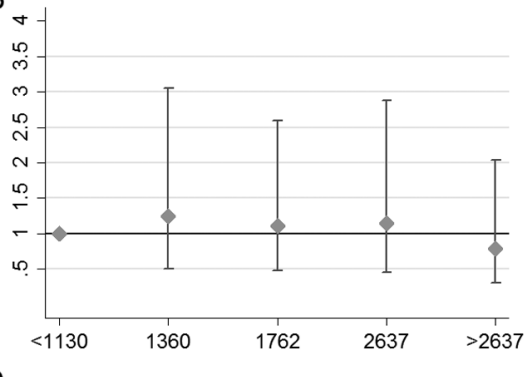

D

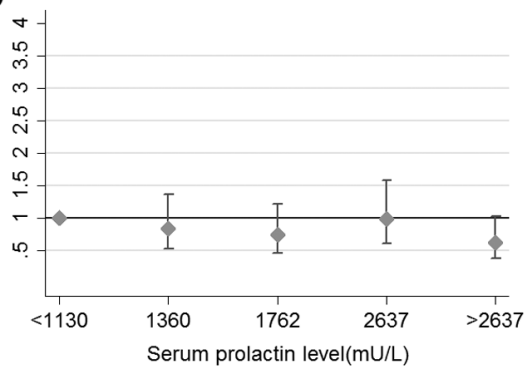

\section{Figure 1}

Estimated HRs $( \pm 95 \% \mathrm{Cl})$ for several clinical outcomes in patients with hyperprolactinaemia according to serum prolactin levels. Footnote: Quintiles of maximum serum prolactin levels (<1130, 1131-1360, 1361-1762, 1763-2637, $>2637$ U/L). Hazard ratios calculated using Cox proportional hazards' models. (A) Diabetes mellitus model adjusted for age and gender. Wald linear test of parameter estimates $\mathrm{Chi}^{2}$ (3) $=3.59, P=0.30$. (B) Non-fatal cardiovascular disease model adjusted for age, gender, history of diabetes mellitus and renal impairment; Wald linear test $\mathrm{Chi}^{2}$ (3) $=0.93, P=0.81$. (C) Bone fractures model adjusted for history of bisphosphonates use, prednisolone use and renal impairment; Wald linear test $\mathrm{Chi}^{2}$ (3) $=0.73$, $P=0.86$. (D) Mortality model adjusted for age, gender, history of non-fatal cardiovascular disease, renal impairment and a Scottish index of multiple deprivation; Wald linear test $\mathrm{Chi}^{2}(3)=3.26, P=0.35$. cohort. The total follow-up was 70,836 person-years with a mean follow-up of 10.1 years (s.D. 6.9) and a maximum follow-up of 25.7 years.

The distribution of patients by age and gender was nearly identical between the two cohorts as expected in a matched design (Table 2). The majority of patients were women $(78 \%)$ and mean age was 39 years. SIMD was unavailable for 2.8 and $4 \%$ of the exposed and unexposed cohorts, respectively. SIMD and serum TSH levels were different between the two cohorts; patients with hyperprolactinaemia were more deprived and had lower TSH values at baseline. Averaged maximum serum concentration of prolactin was much higher in patients with hyperprolactinaemia by definition (1514U/L vs $193 \mathrm{U} / \mathrm{L})$. The exposed cohort was more likely to have diabetes (80\% type 2), cardiovascular disease, bone fractures or infectious conditions and has been prescribed prednisolone as compared to the unexposed cohort at baseline, but was less likely to be prescribed oestrogen-containing hormone replacement therapy. The pituitary disorder group was further stratified into 196 microadenoma and 54 macroadenoma cases based upon linked MRI reports where available.

We evaluated the morbidity risks associated with hyperprolactinaemia by the respective patient subgroup (Table 3). No increased morbidity was observed in any of the patient subgroups except those with drug-induced hyperprolactinaemia where there was an increased risk of diabetes mellitus ( $\mathrm{HR}=1.63,95 \% \mathrm{CI}$ : 1.15-2.32), non-fatal cardiovascular disease $(\mathrm{HR}=1.85,95 \% \mathrm{CI}$ : 1.17-2.93), bone fractures $(\mathrm{HR}=2.08,95 \% \quad \mathrm{CI}: 1.47-2.94)$ and infectious conditions ( $\mathrm{HR}=1.89,95 \% \mathrm{CI}$ : 1.48-2.44) after adjustment for potential confounding baseline characteristics. However, when the increased risks of these adverse health outcomes were analysed by quintiles of prolactin concentration, no significant dose-response relationship was observed (Fig. 1A, B and C). No increased risk of all-cause cancer or breast cancer $(\mathrm{HR}=0.98,96 \%$ CI: 0.56-1.71) was observed in the drug-induced group or any of the other groups.

Finally, we examined mortality risk by subgroup. No increased mortality was observed in patients with pituitary microadenomas. In contrast, increased mortality risks were observed in each of the other subgroups including those with pituitary macroadenomas and those with drug-induced hyperprolactinaemia (Table 3). However, when we evaluated mortality outcomes by quintiles of prolactin concentration, no significant trend was observed either (Fig. 1D) indicating that any relationship between mortality-raised prolactin is unlikely to be causal.

\section{Discussion}

This study demonstrated that patients with hyperprolactinaemia due to pituitary microadenomas and macroadenomas had no increased risk of cardiovascular disease, diabetes, cancer, bone fractures or infectious diseases. The most common treatment for these conditions is the use of dopamine agonists, and the http://www.endocrineconnections.org
DOI: $10.1530 /$ EC-17-0171
() 2017 The authors Published by Bioscientifica Ltd
This work is licensed under a Creative Commons Attribution-NonCommercial 4.0 International License. 
lack of association with adverse endpoints is reassuring. Patients with drug-induced hyperprolactinaemia had an increased risk of diabetes, cardiovascular disease, bone fractures and infectious diseases but not of cancer. We performed a further dose-response analysis (Fig. 1), suggesting that the association between these endpoints and serum prolactin is unlikely to be causal. Of note, antipsychotic agents, which are the commonest group of drugs that cause hyperprolactinaemia, are known to cause weight gain which increases the risk of both diabetes and cardiovascular disease. Our studies indicate that these adverse health outcomes are unlikely to be directly related to the increased serum prolactin concentration.

Patients with pituitary microadenomas had no increased mortality, which is reassuring as this is the common type of pituitary tumour associated with hyperprolactinaemia, whilst patients with hyperprolactinaemia due to macroadenomas, druginduced hyperprolactinaemia and hypothyroidism all had increased mortality. However, we subsequently demonstrated an absence of any dose-response relationship between the extent of serum prolactin elevation and the risk of death, once again indicating that the association with this outcome is unlikely to be direct (i.e. not due to the raised serum prolactin per se). In the current analysis, we have insufficient data to explore the possible reasons for the increased risk of death, although premature mortality has been reported in patients with pituitary macroadenomas. For example, other hormonal deficiencies as a result of hypopituitarism or excessive steroid replacement in the past may contribute to adverse health outcomes (30). Similarly, poorly controlled hypothyroidism, to the level that causes secondary hyperprolactinaemia, has been shown to cause increased death rates (31), whilst the increased death risk for people with drug-induced hyperprolactinaemia may be due to the higher risk of diabetes and cardiovascular disease associated with antipsychotic drugs. Increased risk of suicide could also be expected for people on antipsychotropic drugs. In addition, it is possible that patients with mental health disorders taking antipsychotic drugs are more likely to have adverse lifestyle factors including smoking and/or physical inactivity, not captured in the current study.

It is very reassuring to note that across all the groups of hyperprolactinaemic patients, none were associated with an increased risk of overall cancer or breast cancer. The prospective Nurses' Health Study (NHS and NHSII) and a meta-analysis of 7 observational studies reported an increased risk of breast cancer of borderline significance, with the latter showing a relative risk of 1.16 (95\% CI: 1.04-1.29) for the highest vs lowest levels of serum prolactin $(16,8)$. A small increased overall cancer risk (HR $=1.31$, 95\% CI: 1.02-1.68) was reported by Berinder et al. from a population-based cohort study of 969 patients with visible pituitary adenomas and/or diagnosis of hyperprolactinaemia followed for 14 years but no increased risk of breast cancer was observed (12). However, several large case-controlled and population-based cohort studies have failed to show any increased risk of breast cancer $(9,11,14)$. As in our study, Berinder et al. was not able to adjust for confounders regarding cancer, but we were able to adjust for oestrogen containing hormone replacement therapy regarding breast cancer (12). Our large population-based study adds further support to the lack of association between raised serum prolactin and breast cancer.

Excluding drug-induced causes, none of the hyperprolactinaemia groups were associated with the risk of diabetes. This is notable, as both negative and positive associations between serum prolactin and diabetes risk have been reported. For example, one study demonstrated a negative association between serum prolactin concentrations and risk of diabetes in women (HR=0.48, 95\% CI: 0.26-0.90) (32), whilst others indicated an increased risk of diabetes in men with increasing prolactin concentrations within the reference range (OR=1.70, 95\% CI: 1.04-2.78) (7). Furthermore, in vivo studies have demonstrated that prolactin is a key regulator of $\beta$-cell proliferation in rodents and that lactogenic hormones are responsible for expansion of $\beta$-cell mass during pregnancy (33). Thus, the absence of an overt association between hyperprolactinaemia and diabetes risk in the current clinical study is both important and reassuring. Furthermore, our dose-response analysis indicates that the increased risk of diabetes in the druginduced hyperprolactinaemia group is unlikely to be directly related to serum prolactin concentrations.

High concentrations of serum prolactin have been related to decreased bone mineral density and an increased risk of bone fragility $(25,17,18)$. A $13 \%$ increased rate of fractures was reported in patients prescribed both prolactin-increasing and non-prolactinincreasing antipsychotics (19). The present study showed an increased risk of fractures, but only in the druginduced hyperprolactinaemia subgroup. Unfortunately, we were unable to correct for confounding factors such as physical activity, diet and smoking. Patients with http://www.endocrineconnections.org DOI: 10.1530/EC-17-0171
(๔) 2017 The authors Published by Bioscientifica Ltd
This work is licensed under a Creative Commons Attribution-NonCommercial 4.0 International License. 
hyperprolactinaemia secondary to pituitary tumours will usually have been treated with dopamine agonists, with the aim of lowering serum prolactin, which is likely to negate any increased fracture risk associated with hyperprolactinaemia. In contrast, many patients with drug-induced hyperprolactinaemia will not have had their serum prolactin concentrations lowered, thereby remaining at risk of hypogonadism unless treated with appropriate oestrogen or testosterone replacement, and this might explain the increased incidence of fractures. Similarly, this might account for the increased risk of infectious disease in the drug-induced patient group, possibly through an effect on immunomodulation (23).

Limitations of this study include that although results were adjusted for several potential confounders, it was not possible to adjust for body mass or smoking which may have varied between certain groups (e.g. those on antipsychotropic drugs for mental health disorders). Patients considered as exposed in the study included those with a serum prolactin greater than $1000 \mathrm{U} / \mathrm{L}$ and although this cut-off may have missed some patients with more modest clinically relevant prolactin elevations, it excluded patients with transiently raised levels and/or those with stress-induced hyperprolactinaemia. We do not have data on the total duration of exposure to medication in the drug-induced group and patients were classified in this group if they had a record of being prescribed a drug(s) known to elevate prolactin levels within 6 months before and 1 month after the assessment of serum prolactin measurement. It is possible that some of the pituitary macroadenomas may have been due to the stalk effect of non-functioning pituitary macroadenomas, and such patients may have been hypopituitary taking other hormonal replacements such as corticosteroids. Although data on morbidity relate mainly to hospital admissions and would have missed outpatient events, the prescription data included all outpatient prescriptions and the impact of missing values is considered low. This was an observational study and therefore causal interpretations of risk estimates should be taken carefully.

In conclusion, people with hyperprolactinaemia due to pituitary tumours did not have an increased risk of adverse health outcomes including diabetes, cardiovascular disease, bone fractures, infections or cancer. Similarly, individuals with microprolactinomas did not display increased mortality. In contrast, an increased risk of death was observed in all other groups of patients with hyperprolactinaemia, including those with pituitary macroadenomas, although this association was unlikely to be directly related to the elevation in serum prolactin concentration. A number of adverse health outcomes were observed in individuals with druginduced hyperprolactinaemia, including cardiovascular disease, diabetes and fracture, although again this was unlikely to be causal. Importantly, no increased risk of allcause cancer or breast cancer was observed in any of the hyperprolactinemic subgroups.

\section{Declaration of interest}

The authors declare that there is no conflict of interest that could be perceived as prejudicing the impartiality of the research reported.

\section{Funding}

The study was supported in part by the Clinical Endocrinology Trust (UK Registered Charity Number 288679) and the NHS Tayside Research Endowments. Paul J Newey received funding from the Chief Scientist Office, UK (Fellowship SCAF/15/01).

\section{Author contribution statement}

Enrique Soto-Pedre researched/analysed the data and wrote the manuscript. Graham P Leese planned the study, researched the data and wrote the manuscript. Paul J Newey and John S Bevan contributed to the discussion and reviewed/edited the manuscript.

\section{Acknowledgements}

The authors wish to acknowledge the help of the FARR Institute and the Health Informatics Centre, University of Dundee (Scotland, UK).

\section{References}

1 Melmed S, Casanueva FF, Hoffman AR, Kleinberg DL, Montori VM, Schlechte JA \& Wass JA. Diagnosis and treatment of hyperprolactinemia: an Endocrine Society clinical practice guideline. Journal of Clinical Endocrinology and Metabolism 201196 273-288. (doi:10.1210/jc.2010-1692)

2 Soto-Pedre E, Newey PJ, Bevan JS, Greig N \& Leese GP. The epidemiology of hyperprolactinaemia over 20 years in the Tayside region of Scotland: the Prolactin Epidemiology, Audit and Research Study (PROLEARS). Clinical Endocrinology 201786 60-67. (doi:10.1111/cen.13156)

3 Bernard V, Young J, Chanson P \& Binart N. New insights in prolactin: pathological implications. Nature Reviews Endocrinology 201511 265-275. (doi:10.1038/nrendo.2015.36)

4 Steffensen C, Maegbaek ML, Laurberg P, Andersen M, Kistorp CM, Norrelund H, Sorensen HT \& Jorgensen JO. Heart valve disease among patients with hyperprolactinemia: a nationwide population-based cohort study. Journal of Clinical Endocrinology and Metabolism 201297 1629-1634. (doi:10.1210/jc.2011-3257)

5 Carrero JJ, Kyriazis J, Sonmez A, Tzanakis I, Qureshi AR, Stenvinkel P, Saglam M, Stylianou K, Yaman H, Taslipinar A, et al. Prolactin levels, endothelial dysfunction, and the risk of cardiovascular events and mortality in patients with CKD. Clinical Journal of the American Society of Nephrology 20127 207-215. (doi:10.2215/CJN.06840711)

6 Haring R, Friedrich N, Volzke H, Vasan RS, Felix SB, Dorr M, Meyer Zu Schwabedissen HE, Nauck M \& Wallaschofski H. Positive association
This work is licensed under a Creative Commons Attribution-NonCommercial 4.0 International License. 
of serum prolactin concentrations with all-cause and cardiovascular mortality. European Heart Journal 201435 1215-1221. (doi:10.1093/ eurheartj/ehs233)

7 Therkelsen KE, Abraham TM, Pedley A, Massaro JM, Sutherland P, Hoffmann U \& Fox CS. Association between prolactin and incidence of cardiovascular risk factors in the Framingham Heart Study. Journal of the American Heart Association 20165 e002640. (doi:10.1161/ JAHA.115.002640)

8 Tworoger SS \& Hankinson SE. Prolactin and breast cancer etiology: an epidemiologic perspective. Journal of Mammary Gland Biology and Neoplasia 200813 41-53. (doi:10.1007/s10911-008-9063-y)

9 Tworoger SS, Rosner BA, Willett WC \& Hankinson SE. The combined influence of multiple sex and growth hormones on risk of postmenopausal breast cancer: a nested case-control study. Breast Cancer Research 201113 R99. (doi:10.1186/bcr3040)

10 Tworoger SS, Eliassen AH, Zhang X, Qian J, Sluss PM, Rosner BA \& Hankinson SE. A 20-year prospective study of plasma prolactin as a risk marker of breast cancer development. Cancer Research 201373 4810-4819. (doi:10.1158/0008-5472.CAN-13-0665)

11 Dekkers OM, Romijn JA, de Boer A \& Vandenbroucke JP. The risk for breast cancer is not evidently increased in women with hyperprolactinemia. Pituitary 201013 195-198. (doi:10.1007/s11102009-0214-y)

12 Berinder K, Akre O, Granath F \& Hulting AL. Cancer risk in hyperprolactinemia patients: a population-based cohort study. European Journal of Endocrinology 2011165 209-215. (doi:10.1530/EJE11-0076)

13 Stattin P, Rinaldi S, Stenman UH, Riboli E, Hallmans G, Bergh A \& Kaaks R. Plasma prolactin and prostate cancer risk: a prospective study. International Journal of Cancer 200192 463-465. (doi:10.1002/ijc.1191)

14 Dekkers OM, Ehrenstein V, Bengtsen M, Farkas DK, Pereira AM, Sorensen HT \& Jorgensen JO. Breast cancer risk in hyperprolactinemia: a population-based cohort study and metaanalysis of the literature. European Journal of Endocrinology 2015173 269-273. (doi:10.1530/EJE-15-0282)

15 Tikk K, Sookthai D, Fortner RT, Johnson T, Rinaldi S, Romieu I, Tjonneland A, Olsen A, Overvad K, Clavel-Chapelon F, et al. Circulating prolactin and in situ breast cancer risk in the European EPIC cohort: a case-control study. Breast Cancer Research 20151749. (doi:10.1186/s13058-015-0563-6)

16 Wang M, Wu X, Chai F, Zhang Y \& Jiang J. Plasma prolactin and breast cancer risk: a meta- analysis. Scientific Reports 2016625998. (doi:10.1038/srep25998)

17 Bolanowski M, Zadrozna-Sliwka B, Jawiarczyk A \& Syrycka J. The influence of other than prolactin hormones on bone mineral density in women with hyperprolactinaemia of various origins. Gynecological Endocrinology 201026 623-627. (doi:10.3109/09513591003686320)

18 Mazziotti G, Porcelli T, Mormando M, De Menis E, Bianchi A, Mejia C, Mancini T, De Marinis L \& Giustina A. Vertebral fractures in males with prolactinoma. Endocrine 201139 288-293. (doi:10.1007/s12020011-9462-5)

19 Sorensen HJ, Jensen SO \& Nielsen J. Schizophrenia, antipsychotics and risk of hip fracture: a population-based analysis. European
Neuropsychopharmacology 201323 872-878. (doi:10.1016/ j.euroneuro.2013.04.002)

20 Allen SH, Sharp GC, Wang G, Conley C, Takeda Y, Conroy SE \& Walker SE. Prolactin levels and antinuclear antibody profiles in women tested for connective tissue disease. Lupus 1996 5 30-37.

21 Notsu K, Ito Y, Furuya H, Ohguni S \& Kato Y. Incidence of hyperprolactinemia in patients with Hashimoto's thyroiditis. Endocrine Journal 199744 89-94. (doi:10.1507/endocrj.44.89)

22 Harirchian MH, Sahraian MA \& Shirani A. Serum prolactin level in patients with multiple sclerosis: a case control study. Medical Science Monitor 200612 CR177-CR180.

23 Orbach H, Zandman-Goddard G, Boaz M, Agmon-Levin N, Amital H, Szekanecz Z, Szucs G, Rovensky J, Kiss E, Doria A, et al. Prolactin and autoimmunity: hyperprolactinemia correlates with serositis and anemia in SLE patients. Clinical Reviews in Allergy and Immunology 201242 189-198.

24 Karimifa M, Tahmasebi A, Bonakdar ZS \& Purajam S. Correlation of serum prolactin levels and disease activity in systematic lupus erythematosus. Rheumatology International 201333 511-516. (doi:10.1007/s00296-011-2211-5)

25 Bolanowski M, Halupczok J \& Jawiarczyk-Przybylowska A. Pituitary disorders and osteoporosis. International Journal of Endocrinology 2015 2015 206853. (doi:10.1155/2015/206853)

26 De Hert M, Detraux J \& Stubbs B. Relationship between antipsychotic medication, serum prolactin levels and osteoporosis/osteoporotic fractures in patients with schizophrenia: a critical literature review. Expert Opinion on Drug Safety 201615 809-823. (doi:10.1517/ 14740338.2016.1167873)

27 ISD Scotland. Data quality assurance; assessments of SMR. Edinburgh, UK: ISD Scotland, 2015. (available at: http://www.isdscotland.org/ Products-and-Services/Data-Quality/Assessments/)

28 Grambsch PM. Goodness-of-fit and diagnostics for proportional hazards regression models. Cancer Treatment and Research $1995 \mathbf{7 5}$ 95-112.

29 Mamtani R, Haynes K, Finkelman BS, Scott FI \& Lewis JD. Distinguishing incident and prevalent diabetes in an electronic medical records database. Pharmacoepidemiology and Drug Safety 2014 23 111-118. (doi:10.1002/pds.3557)

30 Jasim S, Alahdab F, Ahmed AT, Tamhane S, Prokop LJ, Nippoldt TB \& Murad MH. Mortality in adults with hypopituitarism: a systematic review and meta-analysis. Endocrine 201656 33-42. (doi:10.1007/ s12020-016-1159-3)

31 Akirov A, Gimbel H, Grossman A, Shochat T \& Shimon I. Elevated TSH in adults treated for hypothyroidism is associated with increased mortality. European Journal of Endocrinology 2017176 57-66. (doi:10.1530/EJE-16-0708)

32 Wang T, Xu Y, Xu M, Ning G, Lu J, Dai M, Xu B, Sun J, Sun W, Lai S, et al. Circulating prolactin and risk of type 2 diabetes: a prospective study. American Journal of Epidemiology 2016184 295-301. (doi:10.1093/aje/kwv326)

33 Banerjee RR, Cyphert HA, Walker EM, Chakravarthy H, Peiris H, Gu X, Liu Y, Conrad E, Goodrich L, Stein RW, et al. Gestational diabetes mellitus from inactivation of prolactin receptor and MafB in islet beta-cells. Diabetes 201665 2331-2341. (doi:10.2337/db15-1527)

Received in final form 24 August 2017

Accepted 12 September 2017 http://www.endocrineconnections.org DOI: 10.1530/EC-17-0171
() 2017 The authors Published by Bioscientifica Ltd

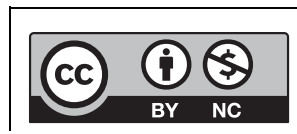

This work is licensed under a Creative Commons Attribution-NonCommercial 4.0 International License. 